\title{
Dynamic Network Slice Resources Reconfiguration in Heterogeneous Mobility Environments
}

\author{
Flavio Meneses $^{1 *}$ | Rui Silva ${ }^{1}$ | Daniel Corujo ${ }^{1}$ | Augusto Neto ${ }^{2}$ | Rui L. Aguiar ${ }^{1}$ \\ ${ }^{1}$ Instituto de Telecomunicações and Universidade de Aveiro, Aveiro, Portugal \\ ${ }^{2}$ Universidade Federal do Rio Grande do Norte (UFRN), Natal-RN, Brazil \\ *Correspondence: flaviomeneses@av.it.pt
}

\begin{abstract}
This paper proposes a framework that optimizes network slicing provisioning in over-the-top (OTT) scenarios, by reducing occupied resources of slices from where the User Equipment (UE) handovers from. To achieve this, the framework leverages an existing Software Defined Networking (SDN)-based UE virtualisation scheme, which allows to instantiate in the cloud a representation of the physical device and its current connectivity context. This scheme was extended with the ability to anchor the UE's datapath and mask the underlying slices in a single end-to-end slice, allowing handover mechanisms between slices to become transparent to involved endpoints. This framework was implemented and evaluated in an experimental testbed where the UE moves between Wi-Fi and LTE slices, with results showing that upstream and downstream throughput is dynamically adapted to the UE requirements prior to the handover.
\end{abstract}

Keywords: Network Slicing, NFV, SDN, OpenFlow, Handover, Mobile offloading, Heterogeneous.

Personal use of this material is permitted. Permission from Wiley must be obtained for all other uses, in any current or future media, including reprinting/republishing this material for advertising or promotional purposes, creating new collective works, for resale or redistribution to servers or lists, or reuse of any copyrighted component of this work in other works. This is the final submitted copy of a paper accepted for publication in the Internet Technology Letters journal.

\section{INTRODUCTION}

The user traffic patterns have been changing along with the network evolution. As the network evolves to better support traffic demands and new services emerge, users have been exploiting the increasing capabilities of wireless user equipments (UE) resulting in highly dynamically communications (i.e., ranging from a voice call to a ultra-high definition video). In fact, users already consume more video traffic on their mobile devices than any other type of data traffic [1]. Moreover, UEs, such as current smartphones, are able to be simultaneously connected to multiple wireless access networks (e.g., LTE and Wi-Fi) while on the move. This not only allows the communication through different access technologies, but also creates a challenge in terms of traffic and mobility management.

In parallel, mobile network operators (MNOs) and Internet service providers (ISP) have been looking for ways to optimise their infrastructure usage in terms of resources. Network slicing 
promises to dynamically partition the network in multiple logically isolated slices, allowing infrastructure and resource sharing[2], and consequently operators optimise resource usage by dynamically increasing (or decreasing) the capabilities of their network. Network Function Virtualisation (NFV) and Software Defined Networking (SDN) have been the preferred adopted technologies to develop such network architecture. NFV allows network functions (NFs) to be instantiated in generic hardware in data-centers. Complementary, SDN allows the dynamic reconfiguration of the datapath via southbound (SB) interfaces[3], such as the OpenFlow (OF) protocol[4]. Also, SDN northbound (NB) interfaces, such as the Representational State Transfer (REST), allows the development of SDN applications to assist the SDN controller in the management of the network. In this context, the proposed elasticity of slicing and the flexibility provided by SDN and NFV technologies, pushes the necessity of developing mechanisms to manage the UE's connectivity while transparently optimise their network resource usage, in order to provide the best user experience (UE) without over-allocating unnecessary resources.

Projects, such as the OpenAirInterface (OAI) [5], implement an open-source 3GPP network, where the NFs of the evolved packet core (EPC) are deployed as virtual NFs (VNFs). FlexRAN [6] extends such framework by allowing the instantiation of multiple 3GPP radio slices. Nevertheless, despite efforts from MNOs to offload data to non-3GPP networks, mechanisms fully supporting seamless handover over heterogeneous networks are still not widespread, specially when considering the elasticity proposed by network slicing. In this line, the virtualisation of the UE's wireless context in the cloud (i.e., vUE), while using OF for flow-based handover have been previously proposed [7, 8]. These mechanisms allow operators and providers to realize enhanced mobility management procedures directly at the cloud, by taking advantage of the UE context existing therein. This mechanism was later enhanced [9] to allow the use of 3GPP network slices and its optimisation considering the current UE's data demands, and offload data from the mobile network into non-3GPP networks, while maintaining connectivity requirements.

This paper further enhances such capabilities by proposing mechanisms to conserve slice resources usage, by reducing resources consumed by network slices that are no longer in use after a UE handovers from them. For this, the OTT uses the vUE to provide key performance indicator (KPI) to the MNO and to anchor its physical counterpart's datapath in the cloud, masking underlying slices in an end-to-end slice. This results in an SDN-based inter-technology handover transparent to the end-points. An experimental evaluation was done considering a LTE to Wi-Fi handover use case, assessing the upstream/downstream throughput and inter-slice handover delay impact, showcasing that the mechanisms do not impact these metrics.

The remainder of the paper is organised as follows. Section $\Pi$ presents the network architecture, followed by the high-level sequence messages for a deployment scenario. The proof-of-concept implementation details and its evaluation are discussed in Section III The paper concludes in Section IV]

\section{Architecture Overview Solution}

Our proposal focuses on network slice optimisation for the highly variable UE's data traffic. For this, the OTT virtualises the UE connectivity context in the cloud, and communicates with telecommunication operators (MNO and ISP) to update the UE's connectivity status and current data requirements. In addition, the OTT uses the vUE to anchor the UE to the network and mask underlying radio and network slices into a end-to-end slice. Here, connectivity context is defined as the UE's current link conditions, active access technologies and surrounding (e.g., neighbour cells). Figure 1 illustrates the network architecture proposal. Next, its building blocks, followed by the high-level messages sequence and a deployment scenario are presented. 


\section{i. Network Architecture}

The network architecture of our proposal is divided in three different providers (MNO, ISP and OTT). The MNO provides a 3GPP core and radio network, with NFs virtualised in cloud, and is capable of creating multiple slices of its network. In this line, the MNO uses an SDN controller to (re)configure the datapath of the network, and an SDRAN controller to manage the wireless slices instantiated in the eNB. Following the 3GPP standards, the Home Subscriber Server (HSS) contains the user profiles and performs the authentication and authorization of the UE's, while the Mobile Entity Management (MME) (de)activates bearers and selects the initial Serving Gateway (SGW). Since, in our proposal, the MNO is SDN-NFV based, the SGW control functions are implemented as SDN applications running along with in the MNO's SDN controller. Thus, the SGW datapath is represented as regular virtual Gateway (vGW).

The ISP illustrates a separate provider which, in this case, provides a non-3GPP access network (e.g., Wi-Fi). Here, the wireless access point (AP) manages Layer 2 (L2) services (i.e., attachment and association), while services such as firewall are implemented in cloud by the ISP's vGW. Consequently, the vGW can manage the L3 services of multiple APs. Also, an ISP's SDN controller allows the ISP to dynamically (re)configure its network, and to instantiate and manage Wi-Fi slices in the AP.

The OTT represents a third-party providing a service that ensures the optimal connection and QoE for its UE, by negotiating with the MNO and OTT to ensure the UE's communication requirements and the optimal network slice resource allocation. Nowadays this role is deployed in entities existing at the MNO, but, in our paper, we exploit the flexible capabilities of SDN and NFV allowing such new players to appear in the market in the future. For this, the OTT virtualises the UE's connectivity context in the cloud (i.e., the vUE), while anchoring its datapath in the OTT's vGW. The context updater not only is responsible for optimising slice attached to the UE, by negotiating with operators and providing KPIs (e.g., current flows and data demands), but also assists in the vUE update.

In our proposal, the UE is enhanced with SDN capabilities, allowing its wireless connectivity to be remotely managed by its virtual counterpart. Moreover, this allows the UE to directly communicate with its virtual counterpart and, upon network event triggers (e.g., connection up, going down or lost, etc.), to be assisted in the handover of current flows to the optimal network alternative. Thus, the vUE monitors its physical counterpart's connectivity, and when a handover or slice optimisation opportunity occurs, the vUE reconfigures its physical counterpart's datapath while requesting (through the context updater) a slice optimisation to the telecommunication.

\section{ii. Deployment scenario and high-level messages sequence}

A proof-of-concept implementation was deployed in an in-house testbed and tested for TCP and UDP data traffic, for both upload and download streams. The control signalling for both protocols and stream direction are similar, and illustrated in Figure 2. With the purpose of facilitating the procedures flow, Figure 2 presents the core networks of the three providers as single entity. In this context, the internal control signalling of each provider is out-of-scope of this work [8, 9].

Initially the UE is connected to the LTE network, and downloading (or uploading) data (1). Passing by an AP, the UE attaches to the non-3GPP network (2) and informs its virtual counterpart via an OF packet_in message (3). In turn, if it results in an handover policy, the vUE updates the flow tables of its physical counterpart (4) and in the vGW (via context updater) via OF flow_modification messages. When the handover is completed, the OTT (through the context updater and via REST) notifies the MNO (5), which optimises the wireless resources of 3GPP slices, by moving the UE for a slice with less capabilities. Since the UE's datapath is anchored 


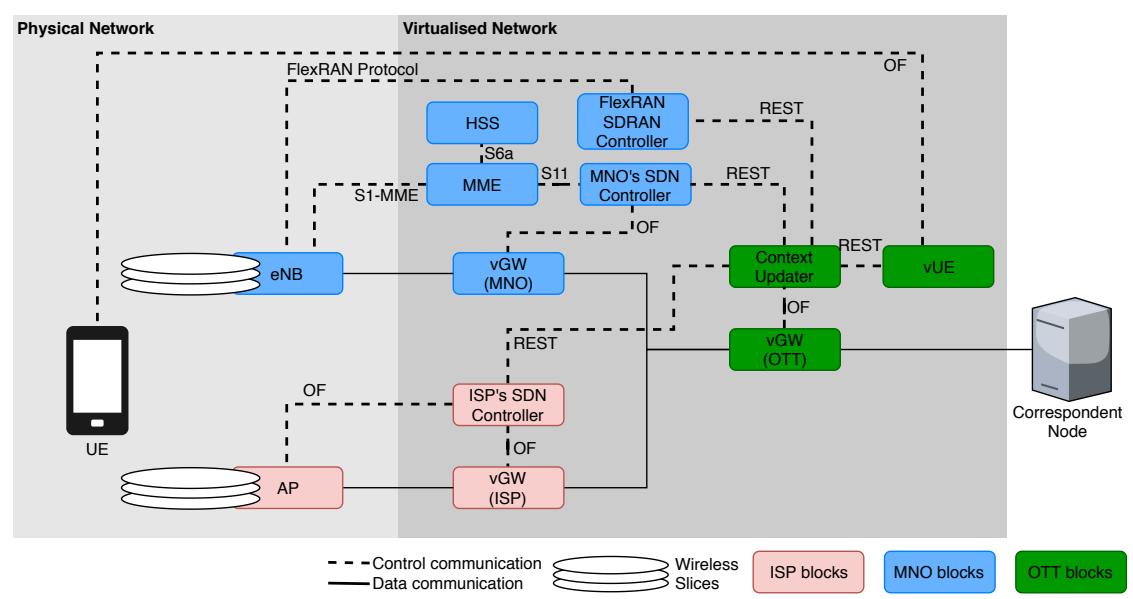

Figure 1: Network architecture and building blocks.

in the OTT network by the vUE, masking underlying slices in a end-to-end slice, the handover process becomes transparent and seamless to both the UE and the $\mathrm{CN}$ (6).

Moving away from the AP, the link strength to the non-3GPP reaches a minimum threshold and the UE informs its virtual counterpart (7). In turn, the OTT notifies the MNO (8), allowing it to prepare and optimise the UE's slice requirements to receive the data by moving it to a slice with more capabilities. After switching the UE's attached slice, the MNO informs the OTT, which in turn updates the flow tables of the UE and vGW (9) via OF flow_modification messages. Again, as the UE's datapath is anchored in the OTT's network, underlying slices are masked in a end-to-end slice and the handover procedures become transparent to the UE and CN (10). As result, the OTT is able to communicate with telecommunication operators and dynamically handover the UE's data among heterogeneous wireless slices with the purpose of guaranteeing the optimal UE's connection and wireless resource allocation.

\section{Proof-of-Concept Implementation and Evaluation}

As previously stated, our architecture aims the cooperation among different providers (i.e., $\mathrm{MNO}$, ISP and OTT). For this the OTT virtualises the UE wireless context and uses the acquired context for negotiating the optimal slice capabilities with telecommunication operators. Interactions among providers are performed through SDN NB applications for acquiring context from both radio and core networks and optimising slices considering current UE's data requirements. The architecture proposal was implemented in an experimental testbed, and evaluated in the scenario presented in the previous section. Next we present the implementation details of our architecture, followed by the its evaluation.

\section{i. Implementation and Deployment}

The UE is a dual-interfaced wireless device able to connected to 3GPP and non-3GPP networks while on the move, similarly to nowadays' smartphones. To allow the UE's connectivity to be remotely and dynamically managed by its virtual counterpart, the Open vSwitch (OvS) was deployed therein with a bridge per interface[9]. The UE was deployed in a PC Engines' APU2C4 with the Huawei ME909 and the wle600vx, wireless modules, and running the Ubuntu server 


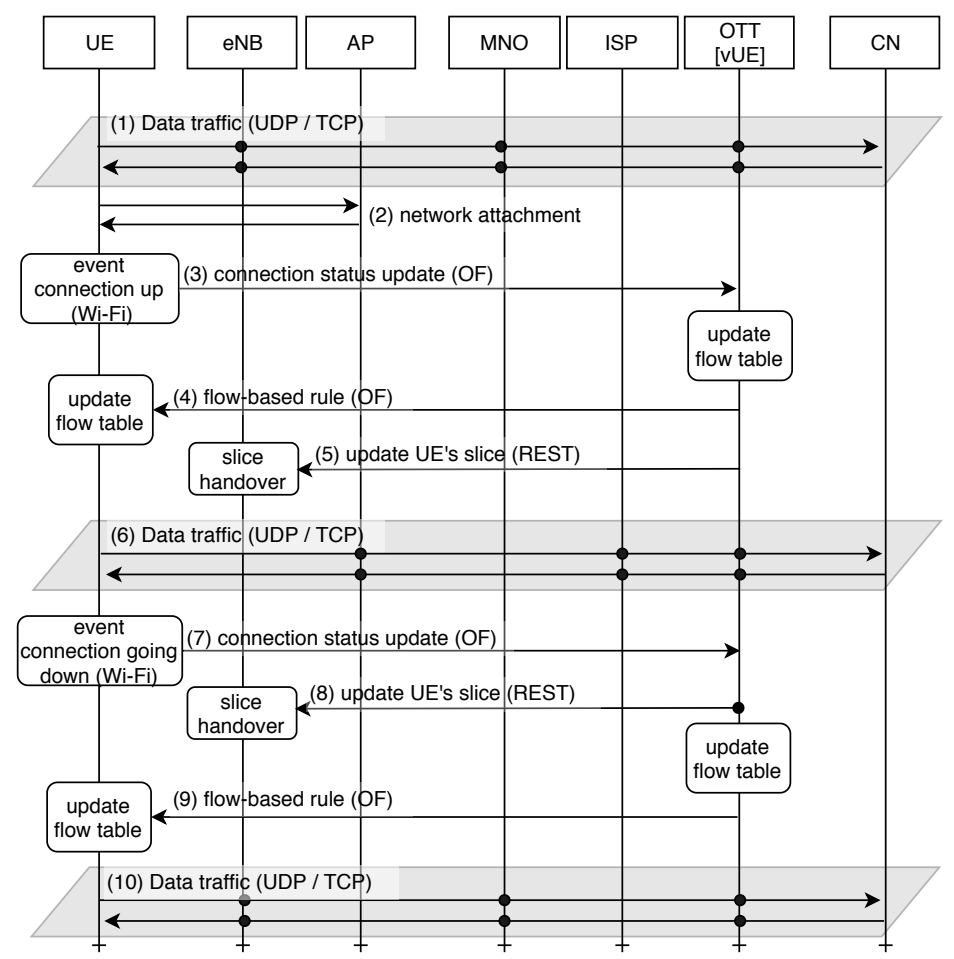

Figure 2: High-level message sequence for the deployment scenario.

\subsection{LTS operating system (OS).}

The MNO provides 3GPP access to the UE. Our deployment was based in OAI project, and EPC's NFs were deployed in virtual machines (VMs), while the eNB was deployed in physical machine running Ubuntu desktop 16.04 LTS OS with an Intel Core i7-7700K CPU and 32 GB of RAM, and an USRP B210 software-defined radio (SDR) board with a LP0965 antenna. To extend the radio access network (RAN) with wireless slicing capabilities, the FlexRAN SDRAN controller was deployed.

Regarding to the ISP, it provides non-3GPP wireless access to the UE via Wi-Fi. The AP's wireless and slicing capabilities were implemented using hosatpd software. To allow the AP's dynamic reconfiguration by ISP's SDN controller, the OvS was installed therein for datapath re-configuration, and an API was developed to allow the reconfiguration of the hostapd software and its slices. The UE's data is re-directed to the ISP's vGW, where L3 services, such as firewall, are implemented. The vGW then re-directs the traffic to the respective OTT. The AP was deployed in PC Engines' APU2C4 with the wle600vx Wi-Fi module and running the Ubuntu server 14.04 LTS OS. The AP was configured in the IEEE 802.11n standard at $5 \mathrm{GHz}$.

Finally, MNO's and ISP's core network, and OTT's NFs (i.e., vGW, context updater and vUE) were deployed in an in-house data-centre running OpenStack (Ocata), in VMs with 1 vCPU, 2 GB of RAM and Ubuntu 16.04 LTS OS. Both the context updater and vUE were implemented as SDN applications. 


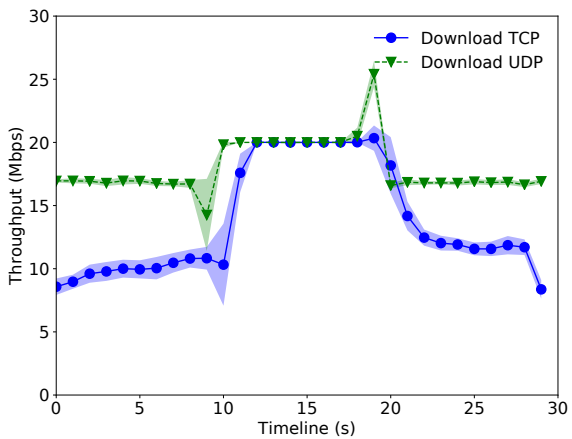

(a) Download data throughput over time.

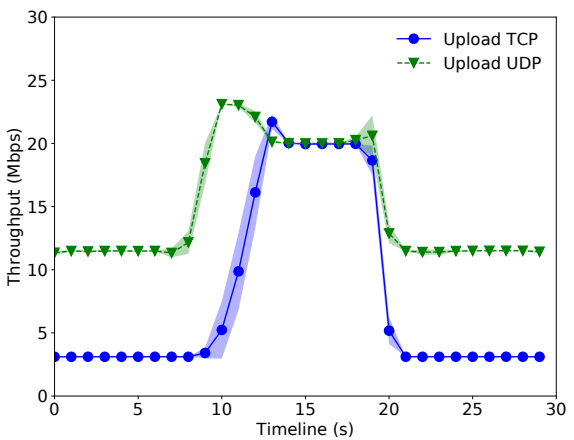

(b) Upload data throughput over time.

Figure 3: Experimental results of the architecture proposal.

\section{ii. Discussion and Evaluation}

Our architecture was evaluated under the scenario presented in section ii The experiments were run 25 times, with the results presenting its average with a confidence interval of $95 \%$. Figure 3 illustrates the download and upload throughputs for both wireless networks. Also, the 3GPP network was divided in 2 slices, in order to switch the UE considering its current data communication requirements. The LTE slice $A$ was configured with $25 \%$ of the total bandwidth (approximately $3.5 \mathrm{Mbps}$ of bandwidth). Contrary, slice B was configured with $70 \%$, accomplishing approximately $10.5 \mathrm{Mbps}$. Regarding to $\mathrm{Wi}-\mathrm{Fi}$, it allows approximately $50 \mathrm{Mbps}$, however the instantiated with Wi-Fi slice was set to $20 \mathrm{Mbps}$. Finally, evaluated streams were generated using iperf3 network tool.

Figure 3 a illustrates the throughput over time for TCP and UDP data when a user was downloading data. In the figure, it is noted that both slices maximum allowed bandwidth was accomplished, and as the UE moved to the Wi-Fi slice (at 10s), the throughput increased from $10 \mathrm{Mbps}$ (or $15 \mathrm{Mbps}$ when UDP) to $20 \mathrm{Mbps}$. On the background, as the UE offloads the data to the Wi-Fi slice, the OTT notifies the MNO, which in turn optimises its wireless resources by switching the UE to a slice with less priority and bandwidth (i.e., LTE slice A). Before disconnecting from the Wi-Fi slice (at 20s), the UE informs the OTT, which in turn notifies the MNO that the UE soon will require an higher bandwidth. When the UE moved back to the LTE, it was already attached to slice $B$.

Finally, the throughput for uploading data is presented in Figure $3 \mathrm{~b}$. Here, the throughput behaviour was similar to the downloading data. Nevertheless, the LTE network offers less bandwidth capacity for upload than for download. As such, the maximum throughput for the LTE slice $B$ was approximately $4 M b p s$ and $11 \mathrm{Mbps}$, for TCP and UDP, respectively. Contrary, the Wi-Fi slice kept its throughput on the pre-established 20Mbps. As in the downloading experiment, the MNO optimises its wireless resources by switching the UE's attached slice, after and before the offloading to the Wi-Fi slice. Finally, the handover, for both experiments, from/to the LTE slice to/from the Wi-Fi slice took approximately $7( \pm 2) m s$ and $50( \pm 14) m s$, on the vUE and the UE, respectively. 


\section{CONCLUSIONS AND FutURE WORK}

This paper proposed an architecture aiming a cooperation among different providers (i.e., OTT, MNO and ISP) in order to optimise wireless slice resources. In this context, we proposed an OTT provider, which virtualises the UE's wireless context and negotiates with telecommunication operators (i.e., MNO and ISP) for slice requirements. For this, the OTT anchors the UE's datapath in its network, masking underlying slices in an end-to-end network slice, since handover procedures become transparent for both end-users. Also, a context updater entity allows the OTT to manage vUEs, while interfacing with MNO's and ISP's management entities (i.e., SDN and SDRAN controllers). As result the proposed architecture allows network slice migration and handover in heterogeneous wireless networks. A proof-of-concept implementation was evaluated in a experimental testbed, with results providing indicators of its feasibility and key aspects for a deployment in production networks. Finally, as future work the OpenFlow protocol should be enhanced for a full integration with the UE, by implementing new OpenFlow messages an integrate wireless connectivity event triggers. Also, more secured mechanisms for the communication among telecommunication operators and OTTs should be developed.

\section{ACKNOWLEDGEMENT}

This work is funded by FCT/MEC through national funds under the project PTDC/EEI-TEL/30685/2017, and by the Integrated Programme of SR\&TD "SOCA" (Ref. CENTRO-01-0145-FEDER-000010), cofunded by Centro 2020 program, Portugal 2020, European Union, through the European Regional Development Fund. The experimental validation of this work included usage of the FlexRAN software from "Mosaic-5G", a EURECOM registered trademark (INPI num. 174357 904).

\section{REFERENCES}

[1] Cisco. Cisco Visual Networking Index: Forecast and Trends, 2017-2022. https://www.cisco $\mathrm{com} / \mathrm{c} / \mathrm{en} / \mathrm{us} / \mathrm{solutions/collateral/service-provider/visual-networking-index-vni/}$ white-paper-c11-741490.html. Online; accessed 28 January 2019.

[2] Matias Richart, Javier Baliosian, Joan Serrat, and Juan-Luis Gorricho. Resource slicing in virtual wireless networks: A survey. IEEE Transactions on Network and Service Management, 13(3):462-476, 2016.

[3] Van-Giang Nguyen, Anna Brunstrom, Karl-Johan Grinnemo, and Javid Taheri. SDN/NFVbased mobile packet core network architectures: A survey. IEEE Communications Surveys $\mathcal{E}$ Tutorials, 19(3):1567-1602, 2017.

[4] Nick McKeown, Tom Anderson, Hari Balakrishnan, Guru Parulkar, Larry Peterson, Jennifer Rexford, Scott Shenker, and Jonathan Turner. OpenFlow: enabling innovation in campus networks. ACM SIGCOMM Computer Communication Review, 38(2):69-74, 2008.

[5] Navid Nikaein, Mahesh K Marina, Saravana Manickam, Alex Dawson, Raymond Knopp, and Christian Bonnet. OpenAirInterface: A flexible platform for 5G research. ACM SIGCOMM Computer Communication Review, 44(5):33-38, 2014.

[6] Xenofon Foukas, Navid Nikaein, Mohamed M Kassem, Mahesh K Marina, and Kimon Kontovasilis. FlexRAN: A flexible and programmable platform for software-defined radio access networks. Proceedings of the 12th International on Conference on emerging Networking EXperiments and Technologies, pages 427-441. ACM, 2016. 
[7] F. Meneses, D. Corujo, C. Guimaraes, and R. L. Aguiar. An Abstraction Framework for Flow Mobility in Multi-technology 5G Environments Using Virtualization and SDN. IEEE Conference on Network Softwarization (NetSoft), pages 1-5, July 2017.

[8] F. Meneses, R. Silva, D. Santos, D. Corujo, and R. L. Aguiar. Using sdn and slicing for data offloading over heterogeneous networks supporting non-3gpp access. IEEE 29th Annual International Symposium on Personal, Indoor and Mobile Radio Communications (PIMRC), pages 1-6, Sep. 2018.

[9] Flávio Meneses, Daniel Corujo, Augusto Neto, and Rui L Aguiar. SDN-based End-to-End Flow Control in Mobile Slice Environments. IEEE Workshop on Mobility Support in Slice-based network control for heterogeneous environments (MOBISLICE) (IEEE NFV-SDN workshops), pages 1-6. IEEE, 2018. 